\title{
Well-posedness Results for Models of Elastomers
}

\author{
Azmy S. Ackleh \\ Department of Mathematics \\ University of Louisiana at Lafayette \\ Lafayette, LA 70504-1010 \\ H.T. Banks and Gabriella A. Pinter \\ Center for Research in Scientific Computation \\ North Carolina State University \\ Raleigh, NC 27965-8205
}

November 8, 2000

\begin{abstract}
Existence and uniqueness of weak solutions are shown for different models of the dynamic behavior of elastomers. The models are based on a nonlinear stress-strain relationship (satisfying a locally Lipschitz and affine domination property) and incorporate hysteretic effects as well. The results provide alternatives to previous theories that required monotonicity assumptions on the nonlinearities. Results with a nonlinear constitutive law and nonlinear internal dynamics are presented for the first time.
\end{abstract}

\section{Introduction}

In this paper we examine the theoretical foundations of a series of models for the dynamic behavior of elastomers (filled rubber-like materials). As outlined in previous papers [6, 7], the basic model describing the longitudinal motion of a viscoelastic bar with the upper end $(x=0)$ fixed and a tip mass on the lower end $(x=\ell)$ is given by 


\section{Report Documentation Page}

Form Approved

OMB No. 0704-0188

Public reporting burden for the collection of information is estimated to average 1 hour per response, including the time for reviewing instructions, searching existing data sources, gathering and maintaining the data needed, and completing and reviewing the collection of information. Send comments regarding this burden estimate or any other aspect of this collection of information,

including suggestions for reducing this burden, to Washington Headquarters Services, Directorate for Information Operations and Reports, 1215 Jefferson Davis Highway, Suite 1204, Arlington

VA 22202-4302. Respondents should be aware that notwithstanding any other provision of law, no person shall be subject to a penalty for failing to comply with a collection of information if it

does not display a currently valid OMB control number.

1. REPORT DATE

08 NOV 2000

4. TITLE AND SUBTITLE

Well-posedness Results for Models of Elastomers

6. $\operatorname{AUTHOR}(\mathrm{S})$

7. PERFORMING ORGANIZATION NAME(S) AND ADDRESS(ES)

North Carolina State University,Center for Research in Scientific

Computation,Raleigh,NC,27695-8205

9. SPONSORING/MONITORING AGENCY NAME(S) AND ADDRESS(ES)
3. DATES COVERED

00-00-2000 to 00-00-2000

5a. CONTRACT NUMBER

5b. GRANT NUMBER

5c. PROGRAM ELEMENT NUMBER

5d. PROJECT NUMBER

5e. TASK NUMBER

5f. WORK UNIT NUMBER

8. PERFORMING ORGANIZATION

REPORT NUMBER

10. SPONSOR/MONITOR'S ACRONYM(S)

11. SPONSOR/MONITOR'S REPORT

NUMBER(S)

12. DISTRIBUTION/AVAILABILITY STATEMENT

Approved for public release; distribution unlimited

13. SUPPLEMENTARY NOTES

14. ABSTRACT

see report

15. SUBJECT TERMS

16. SECURITY CLASSIFICATION OF:

a. REPORT

unclassified

b. ABSTRACT

unclassified

c. THIS PAGE

unclassified
17. LIMITATION OF ABSTRACT
18. NUMBER

OF PAGES

15 19a. NAME OF

RESPONSIBLE PERSON 


$$
\begin{aligned}
& \rho A_{c} u_{t t}-\left(A_{c} \sigma\right)_{x}=0 \quad \text { for } 0<x<\ell \\
& M u_{t t}(t, \ell)+A_{c} \sigma(t, \ell)=M g+f(t) \\
& u(t, 0)=0 \\
& u(0, x)=u_{0} \\
& u_{t}(0, x)=u_{1},
\end{aligned}
$$

where $\rho$ is the mass density, $A_{c}$ is cross-sectional area, $M$ is tip mass, $g$ is gravitational acceleration, $f$ is external force, and $\sigma$ denotes the stress. If there is no tip mass and $A_{c}=\rho=1$, then we can write the system in variational formulation as

$$
\begin{aligned}
& u_{t t}-\sigma_{x}=F(t) \text { in } V^{*} \\
& u(0, x)=u_{0} \in V \\
& u_{t}(0, x)=u_{1} \in H,
\end{aligned}
$$

where we let $V=H_{L}^{1}(0, \ell), H=L^{2}(0, \ell)$ and $F(t)=f(t) \delta_{\ell}(x)$. The crucial modeling question is what type of stress strain relationship best describes the material. In our earlier experimentally based investigations of elastomers we found that a nonlinear constitutive law is needed $([4,5])$, i.e.,

$$
\sigma\left(u_{x}\right)=E u_{x}+g_{e}\left(u_{x}\right)+C_{D} u_{t x}
$$

where the third term on the right side is a first approximation to a damping that these materials exhibit. (For simplicity of presentation here we will later make the assumption that $E=1$.) Comparing the actual experimental data with this model, we demonstrated good agreement for unfilled and lightly filled rubber bars. However, the model is not adequate to describe medium or highly filled elastomers that exhibit significant hysteretic behavior. To account for this property of the material we included a Boltzmann integral term in the constitutive relationship

$$
\sigma\left(u_{x}\right)=E u_{x}+g_{e}\left(u_{x}\right)+C_{D} u_{t x}+\int_{-\infty}^{t} e^{-c_{1}(t-s)} \frac{d}{d s} g_{v}\left(u_{x}, u_{t x}\right) d s .
$$

As described in [5] under specific assumptions on the prehistory of the motion this relationship can be expressed in an equivalent internal variable formulation

$$
\begin{aligned}
\sigma\left(u_{x}\right) & =E u_{x}+g_{e}\left(u_{x}\right)+C_{D} u_{t x}+\varepsilon_{1} \\
\varepsilon_{1_{t}}+c_{1} \varepsilon_{1} & =\frac{d}{d t} g_{v}\left(u_{x}, u_{t x}\right) \\
\varepsilon_{1}(0, x) & =0 .
\end{aligned}
$$

We can think of $\varepsilon_{1}$ as an internal strain variable whose dynamics is described by (1.12)-(1.13). We also note that although these constitutive relationships are expressed in terms of the infinitesimal strain $u_{x}$, the formulation is equivalent to considering finite strains $\varepsilon=u_{x}+\frac{1}{2} u_{x}^{2}$, 
with different nonlinear functions $g_{e}$ and $g_{v}$. The internal variable system (1.11)-(1.13) can be further generalized by considering nonlinear internal dynamics, i.e., (1.11) with

$$
\begin{aligned}
& \varepsilon_{1_{t}}+c_{1} \varepsilon_{1}=\frac{d}{d t} g_{v}\left(u_{x}, u_{t x}\right)+g_{i n}\left(\varepsilon_{1}\right) \\
& \varepsilon_{1}(0, x)=0 .
\end{aligned}
$$

We note that the resulting constitutive law is no longer equivalent to a Boltzmann integral formulation. A further generalization that has proved important for highly filled elastomers involves multiple internal variables. That is, one replaces $\varepsilon_{1}$ in (1.11) by a finite sum $\sum \varepsilon_{j}$ of internal variables $\varepsilon_{j}$ which satisfy systems of the form (1.12)-(1.13) or (1.14)-(1.15).

In this note we consider the well-posedness of the basic model with the three different constitutive laws. Thus we first show that the nonlinear problem with no hysteresis ((1.6)-(1.8) with (1.9)) is well posed under rather general assumptions on the nonlinear function $g_{e}$. Our first result guarantees the existence of a unique local weak solution under a local Lipschitz condition on the nonlinear function. If we impose an additional growth assumption on the nonlinearity, then the weak solution is global (i.e., exists for any time interval $[0, T]$ ). We then show that similar results can be obtained for the nonlinear problem (i.e., nonlinear $g_{e}, g_{v}$ ) with linear internal strain dynamics ((1.6)-(1.8) with (1.11)-(1.13)) and for the nonlinear problem with nonlinear internal strain dynamics ((1.6)-(1.8) with (1.11),(1.14)-(1.15)). The first two problems were previously studied under certain monotonicity and growth assumptions on the nonlinearities $\left(g_{e}\right.$ and $\left.g_{v}\right)$ in [2] and [6], respectively. The results in this paper demonstrate that well-posedness can be achieved under relaxed assumptions and can be extended to the problem with nonlinear internal dynamics. The techniques we use were successfully employed to establish existence-uniqueness of weak solutions for linear evolution equations of second order in $t$ in [8] and for semilinear second order evolution equations where the nonlinear forcing term satisfies a global Lipschitz condition in [9]. In [1] such techniques were extended to study a nonlinear beam equation where the nonlinearity satisfies only a local Lipschitz condition. Arguments for our results concerning the nonlinear problem with no hysteresis are very similar to the ones used in [1]. We give a fairly detailed exposition here in order to be able to easily refer to these as we extend the well-posedness result to the systems with internal variables.

\section{The nonlinear problem with no hysteresis}

In this section we investigate the system

$$
\begin{aligned}
& u_{t t}-C_{D} u_{t x x}-u_{x x}-\left(g_{e}\left(u_{x}\right)\right)_{x}=F(t) \text { in } V^{*} \\
& u(0, x)=u_{0} \in V \\
& u_{t}(0, x)=u_{1} \in H,
\end{aligned}
$$

where $V=H_{L}^{1}(0, \ell) \hookrightarrow H=L^{2}(0, \ell) \hookrightarrow V^{*}, \quad C_{D}>0$ and $E$ has been normalized only for the sake of convenience. We make the following assumptions: 
$\left(A_{g_{e}}\right)$ The nonlinear function $g_{e}$ satisfies the following local Lipschitz condition: let $B_{r}(0)$ denote the ball of radius $r$ centered at 0 in $H$ and for some positive constant $L_{B_{r}}$ we have

$$
\left\|g_{e}(w)-g_{e}(v)\right\| \leq L_{B_{r}}\|w-v\|
$$

for all $w, v \in B_{r}(0)$.

$\left(A_{b_{e}}\right)$ There exist constants $C_{1}, C_{2}$ such that

$$
\left\|g_{e}(v)\right\| \leq C_{1}\|v\|+C_{2},
$$

for every $v \in H$.

$\left(A_{f}\right)$ The forcing term $F$ satisfies

$$
F \in L^{2}\left(0, T ; V^{*}\right) .
$$

We define the space of weak solutions to be

$$
\mathcal{U}(0, T)=\left\{u \in L^{2}(0, T ; V) \mid u_{t} \in L^{2}(0, T ; V), u_{t t} \in L^{2}\left(0, T ; V^{*}\right)\right\},
$$

with norm given by

$$
\|u\|_{\mathcal{U}(0, T)}=\left(\|u\|_{L^{2}(0, T ; V)}^{2}+\left\|u_{t}\right\|_{L^{2}(0, T ; V)}^{2}+\left\|u_{t t}\right\|_{L^{2}\left(0, T ; V^{*}\right)}^{2}\right)^{\frac{1}{2}} .
$$

Definition 2.1 We define $u \in \mathcal{U}(0, T)$ to be a weak solution of (2.16)-(2.18) if it satisfies

$$
\begin{aligned}
& \left\langle u_{t t}, \varphi\right\rangle_{V^{*}, V}+C_{D}\left\langle u_{t x}, \varphi_{x}\right\rangle+\left\langle u_{x}, \varphi_{x}\right\rangle+\left\langle g_{e}\left(u_{x}\right), \varphi_{x}\right\rangle=\langle F, \varphi\rangle_{V^{*}, V} \\
& \text { for every } \varphi \in V
\end{aligned}
$$

and

$$
\begin{aligned}
& u(0, x)=u_{0} \in V \\
& u_{t}(0, x)=u_{1} \in H .
\end{aligned}
$$

Here $\langle\cdot, \cdot\rangle$ denotes the inner product in $H$ while $\langle\cdot, \cdot\rangle_{V^{*}, V}$ represents the usual duality product. We first prove the following local existence theorem:

Theorem 2.1 Under assumptions $\left(A_{g_{e}}\right)$ and $\left(A_{f}\right)$ and for any $u_{0} \in V, u_{1} \in H$, the system (2.19)-(2.21) has a unique weak solution on some interval $\left[0, t^{*}\right]$.

Proof: Let $P$ denote the Hilbert space radial retraction onto the ball in $\mathrm{H}$ centered at $u_{0 x}$ with radius 1 , and let

$$
\hat{g}_{e}(v)=g_{e}(P v) .
$$


Thus $\hat{g}_{e}$ satisfies a global Lipschitz condition

$$
\left\|\hat{g}_{e}(w)-\hat{g}_{e}(v)\right\| \leq L_{B_{\left(1+\left\|u_{0 . x}\right\|\right)}}\|P w-P v\| \leq \tilde{L}\|w-v\|
$$

for every $w, v \in H$ and also

$$
\left\|\hat{g}_{e}(w)\right\| \leq \tilde{L}\|w\|+C
$$

where $C=\left\|g_{e}(0)\right\| \geq 0$.

Now we consider the problem

$$
\begin{aligned}
& u_{t t}-C_{D} u_{t x x}-u_{x x}-\left(\hat{g}_{e}\left(u_{x}\right)\right)_{x}=F(t) \text { in } V^{*} \\
& u(0, x)=u_{0} \in V \\
& u_{t}(0, x)=u_{1} \in H .
\end{aligned}
$$

Let $\left\{\psi_{i}\right\}_{i=1}^{\infty}$ be any linearly independent total subset of $V$. For each $m$ let

$$
V^{m}=\operatorname{span}\left\{\psi_{1}, \psi_{2}, \ldots, \psi_{m}\right\},
$$

and choose $u_{0}^{m}, u_{1}^{m} \in V^{m}$ such that $u_{0}^{m} \rightarrow u_{0}$ in $V$ and $u_{1}^{m} \rightarrow u_{1}$ in $H$ as $m \rightarrow \infty$. We develop the standard Galerkin approximations for the problem (2.25)-(2.27). Let $u^{m}(t)=\sum_{i=1}^{m} C_{i}^{m}(t) \psi_{i}$ be the unique solution of the m-dimensional ordinary differential equation system

$$
\begin{aligned}
& \left\langle u_{t t}^{m}, \psi_{j}\right\rangle_{V^{*}, V}+C_{D}\left\langle u_{t x}^{m}, \psi_{j x}\right\rangle+\left\langle u_{x}^{m}, \psi_{j x}\right\rangle+\left\langle\hat{g}_{e}\left(u_{x}^{m}\right), \psi_{j x}\right\rangle=\left\langle F, \psi_{j}\right\rangle_{V^{*}, V} \\
& u^{m}(0, x)=u_{0}^{m} \\
& u_{t}^{m}(0, x)=u_{1}^{m} .
\end{aligned}
$$

To obtain an a priori estimate we multiply (2.28) by $\frac{d}{d t} C_{j}^{m}(t)$ and sum over $j$ to arrive at

$$
\left\langle u_{t t}^{m}, u_{t}^{m}\right\rangle_{V^{*}, V}+C_{D}\left\langle u_{t x}^{m}, u_{t x}^{m}\right\rangle+\left\langle u_{x}^{m}, u_{t x}^{m}\right\rangle+\left\langle\hat{g}_{e}\left(u_{x}^{m}\right), u_{t x}^{m}\right\rangle=\left\langle F, u_{t}^{m}\right\rangle_{V^{*}, V} .
$$

Thus

$$
\frac{1}{2} \frac{d}{d t}\left\{\left\|u_{t}^{m}(t)\right\|^{2}+\left\|u_{x}^{m}(t)\right\|^{2}\right\}+C_{D}\left\|u_{t x}^{m}\right\|^{2}=-\left\langle\hat{g}_{e}\left(u_{x}^{m}\right), u_{t x}^{m}\right\rangle+\left\langle F, u_{t}^{m}\right\rangle_{V^{*}, V} .
$$

Integrating from 0 to $t$ we obtain

$$
\begin{aligned}
& \left\|u_{t}^{m}(t)\right\|^{2}+\left\|u_{x}^{m}(t)\right\|^{2}+2 C_{D} \int_{0}^{t}\left\|u_{\tau x}^{m}(\tau)\right\|^{2} d \tau=\left\|u_{1}^{m}\right\|^{2}+\left\|u_{0 x}^{m}\right\|^{2}-2 \int_{0}^{t}\left\langle\hat{g}_{e}\left(u_{x}^{m}\right), u_{\tau x}^{m}\right\rangle d \tau \\
& +2 \int_{0}^{t}\left\langle F, u_{\tau}^{m}\right\rangle_{V^{*}, V} d \tau .
\end{aligned}
$$

Using assumption $\left(A_{f}\right)$, the boundedness property (2.24) and standard inequalities, we obtain

$$
\begin{aligned}
\left\|u_{t}^{m}(t)\right\|^{2}+\left\|u_{x}^{m}(t)\right\|^{2}+\nu \int_{0}^{t}\left\|u_{\tau x}^{m}(\tau)\right\|^{2} d \tau & \leq\left\|u_{1}^{m}\right\|^{2}+\left\|u_{0 x}^{m}\right\|^{2}+\frac{\tilde{L}^{2}}{\delta} \int_{0}^{t}\left\|u_{x}^{m}(\tau)\right\|^{2} d \tau \\
& +\frac{1}{\delta} \int_{0}^{t}\|F(\tau)\|_{V^{*}}^{2} d \tau+\frac{C^{2} T}{\delta},
\end{aligned}
$$


where $\delta$ is chosen such that $\nu=2 C_{D}-3 \delta>0$. By applying the Gronwall inequality we can conclude that the sequence $\left\{\left\|u_{x}^{m}\right\|^{2}\right\}$ is bounded. Hence there exists a positive constant $\tilde{C}=\tilde{C}\left(u_{1}, u_{0}, g_{e}, F, T\right)$ independent of $m$ such that

$$
\left\|u_{t}^{m}(t)\right\|^{2}+\left\|u_{x}^{m}(t)\right\|^{2}+\nu \int_{0}^{t}\left\|u_{\tau x}^{m}(\tau)\right\|^{2} d \tau \leq \tilde{C} .
$$

Now we can argue as in $[1,2,3,8]$ that there exists a subsequence, again denoted by $\left\{u^{m}\right\}$, and limit functions $u \in W^{1,2}(0, T ; V)$ and $\tilde{g} \in L^{2}(0, T ; H)$, such that

$$
\begin{aligned}
u^{m} & \rightarrow u \text { weakly in } W^{1,2}(0, T ; V) \\
\hat{g}_{e}\left(u_{x}^{m}\right) & \rightarrow \tilde{g} \text { weakly in } L^{2}(0, T ; H) .
\end{aligned}
$$

Using these convergences we obtain that $u$ satisfies

$$
\begin{aligned}
& \left\langle u_{t t}, \varphi\right\rangle_{V^{*}, V}+C_{D}\left\langle u_{t x}, \varphi_{x}\right\rangle+\left\langle u_{x}, \varphi_{x}\right\rangle+\left\langle\tilde{g}, \varphi_{x}\right\rangle=\langle F, \varphi\rangle_{V^{*}, V} \\
& \text { for every } \varphi \in V \text { and } \\
& u(0, x)=u_{0} \in V \\
& u_{t}(0, x)=u_{1} \in H .
\end{aligned}
$$

We note that as in [1] we have that

$$
\varphi \rightarrow\left\langle u_{t t}, \phi\right\rangle_{V^{*}, V}
$$

is continuous over $\mathcal{D}(0, T ; V)$ equipped with the topology of $L^{2}(0, T ; V)$ and thus by density over $L^{2}(0, T ; V)$. So $u_{t t} \in\left(L^{2}(0, T ; V)\right)^{*}=L^{2}\left(0, T ; V^{*}\right)$ and since we already established that $u \in W^{1,2}(0, T ; V)$ we can conclude that $u \in \mathcal{U}(0, T)$. By [8, Remark 1 , p. 555] this also provides the additional regularity:

$$
u \in C([0, T] ; V), \quad u_{t} \in C([0, T] ; H) .
$$

To conclude that $u$ is indeed a weak solution of (2.25)-(2.27) we need to show that

$$
\left\langle\tilde{g}, \varphi_{x}\right\rangle=\left\langle\hat{g}_{e}\left(u_{x}\right), \varphi_{x}\right\rangle \text { for every } \varphi \in V \text {. }
$$

This is achieved by establishing the strong convergence $u_{x}^{m}(t) \rightarrow u_{x}(t)$ in $H$ as $m \rightarrow \infty$. Let $z^{m}(t)=u^{m}(t)-u(t)$. Using $u_{t}^{m}$ and $u_{t}$ as test functions in (2.28) and (2.38), respectively and integrating, we find

$$
\begin{aligned}
& \left\|z_{t}^{m}(t)\right\|^{2}+\left\|z_{x}^{m}(t)\right\|^{2}+2 C_{D} \int_{0}^{t}\left\|z_{\tau x}^{m}(\tau)\right\|^{2} d \tau=\left\|u_{1}^{m}-u_{1}\right\|^{2}+\left\|u_{0 x}^{m}-u_{0 x}\right\|^{2} \\
& -2 \int_{0}^{t}\left\langle\hat{g}_{e}\left(u_{x}^{m}(\tau)\right)-\tilde{g}(\tau), z_{\tau x}^{m}(\tau)\right\rangle d \tau+2 \int_{0}^{t}\left\langle F(\tau), z_{\tau}^{m}(\tau)\right\rangle_{V^{*}, V} d \tau+X_{m}(t),
\end{aligned}
$$


where

$$
\begin{aligned}
X_{m}(t) & =2\left[-\left\langle u_{t}(t), u_{t}^{m}(t)\right\rangle-\left\langle u_{x}(t), u_{x}^{m}(t)\right\rangle-2 C_{D} \int_{0}^{t}\left\langle u_{\tau x}(\tau), u_{\tau x}^{m}(\tau)\right\rangle d \tau+\left\langle u_{1}, u_{1}^{m}\right\rangle\right. \\
& +\left\langle u_{0 x}, u_{0 x}^{m}\right\rangle-\int_{0}^{t}\left\langle\hat{g}_{e}\left(u_{x}^{m}(\tau)\right), u_{\tau x}(\tau)\right\rangle d \tau-\int_{0}^{t}\left\langle\tilde{g}(\tau), u_{\tau x}^{m}(\tau)\right\rangle d \tau \\
& \left.+2 \int_{0}^{t}\left\langle F(\tau), u_{\tau}(\tau)\right\rangle d \tau\right] .
\end{aligned}
$$

Now

$$
\begin{aligned}
2 \int_{0}^{t}\left\langle\hat{g}_{e}\left(u_{x}^{m}(\tau)\right)-\tilde{g}(\tau), z_{\tau x}^{m}(\tau)\right\rangle d \tau & =2 \int_{0}^{t}\left\langle\hat{g}_{e}\left(u_{x}^{m}(\tau)\right)-\hat{g}_{e}\left(u_{x}(\tau)\right), z_{\tau x}^{m}(\tau)\right\rangle d \tau \\
& +2 \int_{0}^{t}\left\langle\hat{g}_{e}\left(u_{x}(\tau)\right)-\tilde{g}(\tau), z_{\tau x}^{m}(\tau)\right\rangle d \tau
\end{aligned}
$$

and

$$
\begin{aligned}
\left|2 \int_{0}^{t}\left\langle\hat{g}_{e}\left(u_{x}^{m}(\tau)\right)-\hat{g}_{e}\left(u_{x}(\tau)\right), z_{\tau x}^{m}(\tau)\right\rangle d \tau\right| & \leq 2 \int_{0}^{t} \tilde{L}\left\|u_{x}^{m}(\tau)-u_{x}(\tau)\right\|\left\|z_{\tau x}^{m}(\tau)\right\| d \tau \\
& \leq \frac{\tilde{L}^{2}}{\delta} \int_{0}^{t}\left\|z_{x}^{m}(\tau)\right\|^{2} d \tau+\delta \int_{0}^{t}\left\|z_{\tau x}^{m}(\tau)\right\|^{2} d \tau
\end{aligned}
$$

Substituting (2.45) into (2.42), we obtain

$$
\begin{aligned}
& \left\|z_{t}^{m}(t)\right\|^{2}+\left\|z_{x}^{m}(t)\right\|^{2}+\left(2 C_{D}-\delta\right) \int_{0}^{t}\left\|z_{\tau x}^{m}(\tau)\right\|^{2} d \tau \leq\left\|u_{1}^{m}-u_{1}\right\|^{2}+\left\|u_{0 x}^{m}-u_{0 x}\right\|^{2} \\
& +\frac{\tilde{L}^{2}}{\delta} \int_{0}^{t}\left\|z_{x}^{m}(\tau)\right\|^{2} d \tau+\left|X_{m}(t)\right|+\left|Y_{m}(t)\right|,
\end{aligned}
$$

where

$$
Y_{m}(t)=2 \int_{0}^{t}\left\langle\hat{g}_{e}\left(u_{x}(\tau)\right)-\tilde{g}(\tau), z_{\tau x}^{m}(\tau)\right\rangle d \tau+2 \int_{0}^{t}\left\langle F(\tau), z_{\tau}^{m}(\tau)\right\rangle_{V^{*}, V} d \tau
$$

Choosing the same subsequence as before we have $\left\|u_{1}^{m}-u_{1}\right\| \rightarrow 0, \quad\left\|u_{0 x}^{m}-u_{0 x}\right\| \rightarrow 0$. Since $z_{t}^{m} \rightarrow 0$ weakly in $L^{2}(0, T ; V)$ we also have that $\left|Y_{m}(t)\right| \rightarrow 0$. We can argue that $\left|X_{m}(t)\right| \rightarrow 0$ as $m \rightarrow \infty$ for a.e. $t$ in the following way: using the convergences (2.36) and (2.37) in (2.43) we obtain the integrated form of (2.38) with $\varphi=u_{t}$. The fact that $u$ satisfies this equation gives the required result. Now by applying the generalized Gronwall inequality to (2.46) we can see that

$$
\left\|z_{x}^{m}(t)\right\|^{2} \rightarrow 0 \text { for a.e. } t \in[0, T]
$$

and we can conclude that

$$
\hat{g}_{e}\left(u_{x}^{m}\right) \rightarrow \hat{g}_{e}\left(u_{x}\right) \text { strongly in } L^{2}(0, T ; H) .
$$

This guarantees (2.41) and thus $u$ is a weak solution of (2.25)-(2.27).

Uniqueness of the weak solution of (2.25)-(2.27) can be shown in the standard way (e.g., see $[2,3,6,8])$. 
We now prove that $(2.16)-(2.18)$ has a unique weak solution on some interval $\left[0, t^{*}\right]$. By the above remarks we know that the weak solution of $(2.25)-(2.27)$ has the property that $u_{x}$ is continuous in $t$. Thus, there exists $t^{*}$ with $0<t^{*} \leq T$ such that

$$
\left\|u_{x}(t)-u_{0 x}\right\| \leq 1 \text { for all } t \in\left[0, t^{*}\right]
$$

and therefore

$$
\hat{g}_{e}\left(u_{x}(t)\right)=g_{e}\left(u_{x}(t)\right) \text { for all } t \in\left[0, t^{*}\right] .
$$

Hence $u$ is a weak solution of (2.16)-(2.18) on $\left[0, t^{*}\right]$. Uniqueness of the weak solution can again be shown in the standard way. This completes the proof of Theorem 2.1.

Now we use the additional assumption $\left(A_{b_{e}}\right)$ to guarantee the existence of a global weak solution.

Theorem 2.2 Under assumptions $\left(A_{g_{e}}\right),\left(A_{b_{e}}\right),\left(A_{f}\right)$ the system (2.16)-(2.18) admits a unique global weak solution.

Proof: As before we can define Galerkin approximations $u^{m}(t)=\sum_{i=1}^{m} C_{j}(t) \psi_{j}$ to solve (2.28)(2.30) with the nonlinear function $g_{e}$ instead of $\hat{g}_{e}$. By assumption $\left(A_{b_{e}}\right)$ we can develop a similar a priori bound:

$$
\left\|u_{t}^{m}(t)\right\|^{2}+\left\|u_{x}^{m}(t)\right\|^{2}+\nu \int_{0}^{t}\left\|u_{\tau x}^{m}(\tau)\right\|^{2} d \tau \leq \tilde{C}=\tilde{C}\left(u_{0}, u_{1}, F, T, C_{1}, C_{2}\right) .
$$

Thus we can again obtain convergences (2.36)-(2.37). Additionally, as in [2, Lemma 5.1] we can show that

$$
u_{x}^{m}(t) \rightarrow u_{x}(t) \text { weakly in } H .
$$

(The arguments to obtain this convergence in [2] depend only on the a priori bound and the general Arzela-Ascoli Theorem and are independent of the specific assumptions on the nonlinear function.) Thus by the weak lower semicontinuity of the norm in $H$ we obtain that

$$
\left\|u_{x}(t)\right\|^{2} \leq \tilde{C} .
$$

So the proof can be completed exactly as before using the local Lipschitz property of $g_{e}$ in the ball $B \sqrt{\tilde{C}}(0)$ in $H$.

\section{The nonlinear problem with linear internal dynamics}

In this section we consider the system

$$
\begin{aligned}
& u_{t t}-C_{D} u_{t x x}-u_{x x}-\left(g_{e}\left(u_{x}\right)\right)_{x}-\varepsilon_{1 x}=F(t) \text { in } V^{*} \\
& \varepsilon_{1_{t}}+c_{1} \varepsilon_{1}=\frac{d}{d t} g_{v}\left(u_{x}, u_{t x}\right) \\
& \varepsilon_{1}(0)=0 \\
& u(0, x)=u_{0} \in V \\
& u_{t}(0, x)=u_{1} \in H
\end{aligned}
$$


where

$$
g_{v}\left(u_{x}, u_{t x}\right)= \begin{cases}g_{v i}\left(u_{x}\right) & \text { if } u_{t x}>0 \\ g_{v d}\left(u_{x}\right) & \text { if } u_{t x}<0\end{cases}
$$

i.e., the viscoelastic response function is different when the strain is increasing and when it is decreasing. The internal dynamics is interpreted in the sense that

$$
\varepsilon_{1}(t)=\int_{0}^{t} e^{-c_{1}(t-s)} \frac{d}{d s} g_{v}\left(u_{x}, u_{s x}\right) d s .
$$

Integrating (3.53) by parts we obtain

$$
\begin{aligned}
\varepsilon_{1}(t) & =g_{v}\left(u_{x}, u_{t x}\right)-\int_{0}^{t} c_{1} e^{-c_{1}(t-s)} g_{v}\left(u_{x}, u_{s x}\right) d s \\
& +\sum_{k=0}^{K} h\left(t-t_{k}\right) e^{-c_{1}\left(t-t_{k}\right)}(-1)^{k+1}\left[g_{v i}\left(u_{x}\left(t_{k}\right)\right)-g_{v d}\left(u_{x}\left(t_{k}\right)\right)\right]
\end{aligned}
$$

where $h$ is the Heaviside function and $t_{k}, \quad k \geq 1$, are the points where, roughly speaking, $u_{t x}\left(t_{k}\right)=0$ with $t_{0}=0$ (e.g., see [6]). More precisely, as explained in detail in [5], [6] the definition of $g_{v}$ is based on the a priori given set of points $\left\{t_{k}\right\}$ where the value of $g_{v}$ takes alternate values $g_{v i}$ and $g_{v d}$ on successive intervals $\left[t_{k}, t_{k+1}\right)$. That is, in the system formulation (based on experimental data) one is given functions $g_{v i}$ and $g_{v d}$ and a sequence of points $\left\{t_{k}\right\}$ so that $g_{v}$ is defined by the alternating values $g_{v i}, g_{v d}$ on intervals $\left[t_{k}, t_{k+1}\right)$. Thus in essence $g_{v}$ depends on $u_{x}$ and $t$ and not on $u_{t x}$. In what follows we will therefore use the notation $g_{v}\left(u_{x}\right)$.

Definition 3.1 We define $\left(u, \varepsilon_{1}\right) \in \mathcal{U}(0, T) \times L^{2}(0, T ; H)$ to be a weak solution of (3.48)-(3.52) if it satisfies

$$
\begin{aligned}
& \left\langle u_{t t}, \varphi\right\rangle_{V^{*}, V}+C_{D}\left\langle u_{t x}, \varphi_{x}\right\rangle+\left\langle u_{x}, \varphi_{x}\right\rangle+\left\langle g_{e}\left(u_{x}\right), \varphi_{x}\right\rangle+\left\langle\varepsilon_{1}, \varphi_{x}\right\rangle=\langle F, \varphi\rangle_{V^{*}, V} \\
& \text { for every } \varphi \in V \\
& u(0, x)=u_{0} \in V \\
& u_{t}(0, x)=u_{1} \in H
\end{aligned}
$$

and

$$
\begin{aligned}
& \varepsilon_{1}(t)=g_{v}\left(u_{x}(t)\right)-\int_{0}^{t} c_{1} e^{-c_{1}(t-s)} g_{v}\left(u_{x}\right) d s \\
& +\sum_{k=0}^{K} h\left(t-t_{k}\right) e^{-c_{1}\left(t-t_{k}\right)}(-1)^{k+1}\left[g_{v i}\left(u_{x}\left(t_{k}\right)\right)-g_{v d}\left(u_{x}\left(t_{k}\right)\right)\right] .
\end{aligned}
$$

We make similar assumptions on $g_{v i}, g_{v d}$ as on $g_{e}$, namely,

$\left(A_{g_{v}}\right)$ The nonlinear functions $g_{v i}, g_{v d}$ satisfy the following local Lipschitz condition: let $B_{r}(0)$ denote the ball of radius $r$ centered at 0 in $H$ and for some positive constants $L_{B_{r}}^{i}$ and $L_{B_{r}}^{d}$ we have

$$
\left\|g_{v i}(w)-g_{v i}(v)\right\| \leq L_{B_{r}}^{i}\|w-v\|
$$




$$
\left\|g_{v d}(w)-g_{v d}(v)\right\| \leq L_{B_{r}}^{d}\|w-v\|
$$

for all $w, v \in B_{r}(0)$.

$\left(A_{b_{v}}\right)$ There exist constants $C_{1}^{i}, C_{1}^{d}, C_{2}^{i}$ and $C_{2}^{d}$ such that

$$
\begin{gathered}
\left\|g_{v i}(v)\right\| \leq C_{1}^{i}\|v\|+C_{2}^{i}, \\
\left\|g_{v d}(v)\right\| \leq C_{1}^{d}\|v\|+C_{2}^{d},
\end{gathered}
$$

for every $v \in H$.

Theorem 3.1 Under assumptions $\left(A_{g_{e}}\right),\left(A_{g_{v}}\right),\left(A_{f}\right)$ the system (3.48)-(3.52) has a unique local weak solution.

Proof: The proof is essentially the same as the proof of Theorem 2.1, so we just outline the crucial steps. First, as in [6] we consider the interval $\left[0, t_{1}\right]$. Let $P$ denote the Hilbert space radial retraction onto the ball in $\mathrm{H}$ centered at $u_{0 x}$ with radius 1 , and define

$$
\begin{aligned}
\hat{g}_{e}(v) & =g_{e}(P v), \\
\hat{g}_{v i}(v) & =g_{v i}(P v), \\
\hat{g}_{v d}(v) & =g_{v d}(P v) .
\end{aligned}
$$

Thus $\hat{g}_{e}, \hat{g}_{v i}, \hat{g}_{v d}$ satisfy the following global Lipschitz and boundedness properties:

$$
\begin{aligned}
\left\|\hat{g}_{j}(w)-\hat{g}_{j}(v)\right\| & \leq L_{j}\|w-v\|, \quad j=e, v i, v d \\
\left\|\hat{g}_{j}(w)\right\| & \leq C_{1}^{j}\|w\|+C_{2}^{j} .
\end{aligned}
$$

Hence we can consider the problem

$$
\begin{aligned}
u_{t t}- & C_{D} u_{t x x}-u_{x x}-\left(\hat{g}_{e}\left(u_{x}\right)\right)_{x} \\
- & \frac{\partial}{\partial x}\left[\hat{g}_{v}\left(u_{x}\right)-e^{-c_{1} t} \hat{g}_{v}\left(u_{0 x}\right)-c_{1} \int_{0}^{t} e^{-c_{1}(t-s)} \hat{g}_{v}\left(u_{x}\right) d s\right]=F(t) \text { in } V^{*}, \\
& u(0, x)=u_{0}, \\
& u_{t}(0, x)=u_{1} .
\end{aligned}
$$

We develop Galerkin approximations $\left\{u^{m}\right\}$ to (3.64)-(3.66). The additional terms in this system as compared to (2.25)-(2.27) cause no difficulties in obtaining an a priori estimate similar to (2.35) due to the properties (3.63). Thus the convergences (2.36)-(2.37) can be obtained and the strong convergence

$$
u_{x}^{m}(t) \rightarrow u_{x}(t) \text { in } H
$$

can be established. One can also easily observe the additional regularity: $u \in C\left(0, t_{1} ; V\right), u_{t} \in$ $C\left(0, t_{1} ; H\right)$, and also that $\varepsilon_{1} \in C\left(0, t_{1} ; H\right)$. By the continuity property of $u_{x}$ in $t$ there exists $t^{*}>0$ such that for $t \in\left[0, t^{*}\right],\left\|u_{x}(t)-u_{0 x}\right\| \leq 1$. Hence $\hat{g}_{j}\left(u_{x}\right)=g_{j}\left(u_{x}\right), j=e, v i, v d$ on $\left[0, t^{*}\right]$. So $u$ is a weak solution of (3.48)-(3.52) on the interval $\left[0, t^{*}\right]$. If $t^{*}=t_{1}$ it makes sense 
to consider the next interval $\left[t_{1}, t_{2}\right]$, where local existence of a weak solution can be established exactly as before. Uniqueness of the weak solution is shown in the standard way.

The existence of a global weak solution can be guaranteed under additional boundedness assumptions.

Theorem 3.2 Under assumptions $\left(A_{g_{e}}\right),\left(A_{g_{v}}\right),\left(A_{b_{e}}\right),\left(A_{b_{v}}\right),\left(A_{f}\right)$ the system (3.48)-(3.52) admits a unique global weak solution.

Proof: First we consider the interval $\left[0, t_{1}\right]$. We develop Galerkin approximations $\left\{u^{m}\right\}$ for (3.48)-(3.52) as before and by the boundedness properties $\left(A_{b_{e}}\right),\left(A_{b_{v}}\right)$ we can obtain an a priori estimate like (2.47). The crucial step in the proof is that we can establish the convergence

$$
u_{x}^{m}(t) \rightarrow u_{x}(t) \text { weakly in } H,
$$

and thus guarantee that $\left\|u_{x}(t)\right\|^{2} \leq \tilde{C}$. Now the local Lipschitz property of $g_{e}$ and $g_{v}$ can be used

in the ball $B \sqrt{\tilde{C}}(0)$ to yield the strong convergence $u_{x}^{m}(t) \rightarrow u_{x}(t)$ in $H$ as before. Uniqueness can be shown in the standard way, and then the weak solution can be extended to the next intervals $\left[t_{i}, t_{i+1}\right], i \geq 1$.

\section{The nonlinear problem with nonlinear internal dynam- ics}

We consider the system

$$
\begin{aligned}
& u_{t t}-C_{D} u_{t x x}-u_{x x}-\left(g_{e}\left(u_{x}\right)\right)_{x}-\varepsilon_{1 x}=F(t) \text { in } V^{*} \\
& \varepsilon_{1_{t}}+c_{1} \varepsilon_{1}=g_{i n}\left(\varepsilon_{1}\right)+\frac{d}{d t} g_{v}\left(u_{x}, u_{t x}\right) \\
& \varepsilon_{1}(0)=0 \\
& u(0, x)=u_{0} \in V \\
& u_{t}(0, x)=u_{1} \in H
\end{aligned}
$$

where the nonlinear functions $g_{e}, g_{v}$ are as in Section 3. We interpret the internal dynamics in the sense that the internal strain $\varepsilon_{1}$ solves

$$
\begin{aligned}
\varepsilon_{1}(t) & =g_{v}\left(u_{x}(t)\right)+\int_{0}^{t} e^{-c_{1}(t-s)}\left[-c_{1} g_{v}\left(u_{x}\right)+g_{i n}\left(\varepsilon_{1}\right)\right] d s \\
& +\sum_{k=0}^{K} h\left(t-t_{k}\right) e^{-c_{1}\left(t-t_{k}\right)}(-1)^{k+1}\left[g_{v i}\left(u_{x}\left(t_{k}\right)\right)-g_{v d}\left(u_{x}\left(t_{k}\right)\right)\right],
\end{aligned}
$$

where again $h$ is the Heaviside function and $t_{k}, k \geq 1$ are defined as in Section 3. We suppose that the nonlinear functions $g_{e}, g_{v}, g_{i n}$ satisfy global Lipschitz properties: 
$\left(A_{L}\right)$ For some positive constants $L^{e}, L^{v i}, L^{v d}$ and $L^{i n}$ we have

$$
\begin{aligned}
\left\|g_{e}(w)-g_{e}(v)\right\| & \leq L^{e}\|w-v\| \\
\left\|g_{v i}(w)-g_{v i}(v)\right\| & \leq L^{v i}\|w-v\| \\
\left\|g_{v d}(w)-g_{v d}(v)\right\| & \leq L^{v d}\|w-v\| \\
\left\|g_{i n}(w)-g_{i n}(v)\right\| & \leq L^{i n}\|w-v\|
\end{aligned}
$$

for all $w, v \in H$.

We prove the following theorem:

Theorem 4.1 Under assumptions $\left(A_{L}\right),\left(A_{f}\right)$ the system (4.67)-(4.71) has a unique global weak solution .

Proof: Let us first consider the interval $\left[0, t_{1}\right]$. On this interval $g_{v}=g_{v i}$ or $g_{v}=g_{v d}$ depending on the initial conditions and the forcing term. We define the following approximate sequence $\left\{u^{N}, \varepsilon_{1}^{N}\right\}$ : let $\left\{u^{N}, \varepsilon_{1}^{N}\right\}$ be the unique weak solution of the system

$$
\begin{aligned}
& \left\langle u_{t t}^{N}, \varphi\right\rangle_{V^{*}, V}+C_{D}\left\langle u_{t x}^{N}, \varphi_{x}\right\rangle+\left\langle u_{x}^{N}, \varphi_{x}\right\rangle+\left\langle g_{e}\left(u_{x}^{N}\right), \varphi_{x}\right\rangle+\left\langle\varepsilon_{1}^{N}, \varphi_{x}\right\rangle=\langle F, \varphi\rangle_{V^{*}, V} \\
& u^{N}(0, x)=u_{0} \in V \\
& u_{t}^{N}(0, x)=u_{1} \in H
\end{aligned}
$$

and

$$
\varepsilon_{1}^{N}=g_{v}\left(u_{x}^{N}\right)-e^{-c_{1} t} g_{v}\left(u_{0 x}\right)+\int_{0}^{t} e^{-c_{1}(t-s)}\left[-c_{1} g_{v}\left(u_{x}^{N}\right)+g_{i n}\left(\varepsilon_{1}^{N-1}\right)\right] d s
$$

where $\varepsilon_{1}^{0}=0$. The sequence is well-defined since (4.77)-(4.80) is the same as (3.55)-(3.58) except for the known term $\int_{0}^{t} e^{-c_{1}(t-s)} g_{i n}\left(\varepsilon_{1}^{N-1}(s)\right) d s$. It is easy to see that Theorem 3.2 extends to this case as well. Also the assumption $\left(A_{L}\right)$ for $g_{e}$ and $g_{v}$ guarantees that $\left(A_{g_{e}}\right),\left(A_{g_{v}}\right),\left(A_{b_{e}}\right)$ and $\left(A_{b_{v}}\right)$ are satisfied. Our goal is to show that $\left\{u^{N}(t)\right\},\left\{u_{x}^{N}(t)\right\},\left\{\varepsilon_{1}^{N}(t)\right\}$ are Cauchy sequences (uniformly in $t<t_{1}$ ).

Let $\hat{u}^{N}=u^{N}-u^{N-1}$ and $\hat{\varepsilon}_{1}^{N}=\varepsilon_{1}^{N}-\varepsilon_{1}^{N-1}$. Then we have

$$
\left\langle\hat{u}_{t t}^{N}, \varphi\right\rangle+C_{D}\left\langle\hat{u}_{t x}^{N}, \varphi_{x}\right\rangle+\left\langle\hat{u}_{x}^{N}, \varphi_{x}\right\rangle+\left\langle g_{e}\left(u_{x}^{N}\right)-g_{e}\left(u_{x}^{N-1}\right), \varphi_{x}\right\rangle+\left\langle\hat{\epsilon}_{1}^{N}, \varphi_{x}\right\rangle=0
$$

With $\varphi=\hat{u}_{t}^{N}$ this gives

$$
\begin{aligned}
& \frac{1}{2} \frac{d}{d t}\left\{\left\|\hat{u}_{t}^{N}(t)\right\|^{2}+\left\|\hat{u}_{x}^{N}(t)\right\|^{2}\right\}+C_{D}\left\|\hat{u}_{x t}^{N}(t)\right\|^{2} \leq\left(L^{e}+L^{v}\right)\left\|\hat{u}_{x}^{N}(t)\right\|\left\|\hat{u}_{x t}^{N}(t)\right\| \\
& +c_{1} L^{v} \int_{0}^{t}\left\|\hat{u}_{x}^{N}(s)\right\| d s\left\|\hat{u}_{t x}^{N}(t)\right\|+L^{i n} \int_{0}^{t}\left\|\varepsilon_{1}^{N-1}(s)-\varepsilon_{1}^{N-2}(s)\right\| d s\left\|\hat{u}_{t x}^{N}(t)\right\|,
\end{aligned}
$$


where $L^{v}=\max \left\{L^{v i}, L^{v d}\right\}$. Thus,

$$
\begin{aligned}
\left\|\hat{u}_{t}^{N}(t)\right\|^{2}+\left\|\hat{u}_{x}^{N}(t)\right\|^{2}+2 C_{D} \int_{0}^{t} & \left\|\hat{u}_{\tau x}^{N}(\tau)\right\|^{2} d \tau \leq 2\left(L^{e}+L^{v}\right) \int_{0}^{t}\left\|\hat{u}_{x}^{N}(\tau)\right\|\left\|\hat{u}_{\tau x}^{N}(\tau)\right\| d \tau \\
& +2 c_{1} L^{v} \int_{0}^{t} \int_{0}^{\tau}\left\|\hat{u}_{x}^{N}(s)\right\| d s\left\|\hat{u}_{\tau x}^{N}(\tau)\right\| d \tau \\
& +2 L^{i n} \int_{0}^{t} \int_{0}^{\tau}\left\|\varepsilon_{1}^{N-1}(s)-\varepsilon_{1}^{N-2}(s)\right\| d s\left\|\hat{u}_{\tau x}^{N}(\tau)\right\| d \tau
\end{aligned}
$$

We estimate the second term on the right side as

$$
\begin{aligned}
& 2 c_{1} L^{v} \int_{0}^{t} \int_{0}^{\tau}\left\|\hat{u}_{x}^{N}(s)\right\| d s\left\|\hat{u}_{\tau x}^{N}(\tau)\right\| d \tau \leq 2 c_{1} L^{v} \int_{0}^{t}\left\|\hat{u}_{x}^{N}(\tau)\right\| d \tau \int_{0}^{t}\left\|\hat{u}_{\tau x}^{N}(\tau)\right\| d \tau \\
& \leq \frac{c_{1} L^{v}}{\delta_{1}^{2}}\left(\int_{0}^{t}\left\|\hat{u}_{x}^{N}(\tau)\right\| d \tau\right)^{2}+c_{1} L^{v} \delta_{1}^{2}\left(\int_{0}^{t}\left\|\hat{u}_{\tau x}^{N}(\tau)\right\| d \tau\right)^{2} \\
& \leq \frac{c_{1} L^{v} t_{1}}{\delta_{1}^{2}} \int_{0}^{t}\left\|\hat{u}_{x}^{N}(\tau)\right\|^{2} d \tau+c_{1} L^{v} \delta_{1}^{2} t_{1} \int_{0}^{t}\left\|\hat{u}_{\tau x}^{N}(\tau)\right\|^{2} d \tau .
\end{aligned}
$$

The third term can be estimated similarly to yield

$$
\begin{aligned}
2 L^{i n} \int_{0}^{t} \int_{0}^{\tau}\left\|\varepsilon_{1}^{N-1}(s)-\varepsilon_{1}^{N-2}(s)\right\| d s\left\|\hat{u}_{\tau x}^{N}(\tau)\right\| d \tau & \leq \frac{L^{i n} t_{1}}{\delta_{1}^{2}} \int_{0}^{t}\left\|\varepsilon_{1}^{N-1}(\tau)-\varepsilon_{1}^{N-2}(\tau)\right\|^{2} d \tau \\
& +L^{i n} \delta_{1}^{2} t_{1} \int_{0}^{t}\left\|\hat{u}_{\tau x}^{N}(\tau)\right\|^{2} d \tau
\end{aligned}
$$

Hence (4.83) gives

$$
\begin{aligned}
\left\|\hat{u}_{t}^{N}(t)\right\|^{2}+\left\|\hat{u}_{x}^{N}(t)\right\|^{2}+\mu_{1} \int_{0}^{t}\left\|\hat{u}_{\tau x}^{N}(\tau)\right\|^{2} d \tau & \leq \mu_{2} \int_{0}^{t}\left\|\hat{u}_{x}^{N}(\tau)\right\|^{2} d \tau \\
& +\mu_{3} \int_{0}^{t}\left\|\varepsilon_{1}^{N-1}(\tau)-\varepsilon_{1}^{N-2}(\tau)\right\|^{2} d \tau
\end{aligned}
$$

where $\mu_{2}=\frac{L^{e}+L^{v}+c_{1} L^{v} t_{1}}{\delta_{1}^{2}}, \mu_{3}=\frac{L^{i n} t_{1}}{\delta_{1}^{2}}$ and $\delta_{1}$ is chosen such that $\mu_{1}=2 C_{D}-\left(L^{e}+L^{v}\right) \delta_{1}^{2}-$ $c_{1} L^{v} \delta_{1}^{2} t_{1}-L^{i n} \delta_{1}^{2} t_{1}>0$. By the Gronwall inequality we obtain

$$
\left\|\hat{u}_{x}^{N}(t)\right\|^{2} \leq \mu_{4} \int_{0}^{t}\left\|\varepsilon_{1}^{N-1}(\tau)-\varepsilon_{1}^{N-2}(\tau)\right\|^{2} d \tau
$$

with $\mu_{4}=\mu_{3} e^{\mu_{2} t_{1}}$, so

$$
\begin{aligned}
\left\|\hat{u}_{t}^{N}(t)\right\|^{2}+\left\|\hat{u}_{x}^{N}(t)\right\|^{2} & \leq \mu_{2} \int_{0}^{t} \mu_{4} \int_{0}^{\tau}\left\|\varepsilon_{1}^{N-1}(s)-\varepsilon_{1}^{N-2}(s)\right\|^{2} d s d \tau \\
& +\mu_{3} \int_{0}^{t}\left\|\varepsilon_{1}^{N-1}(\tau)-\varepsilon_{1}^{N-2}(\tau)\right\|^{2} d \tau \\
& \leq\left(\mu_{2} \mu_{4} t_{1}+\mu_{3}\right) \int_{0}^{t}\left\|\varepsilon_{1}^{N-1}(\tau)-\varepsilon_{1}^{N-2}(\tau)\right\|^{2} d \tau .
\end{aligned}
$$


Similarly,

$$
\begin{aligned}
& \left\|\hat{\varepsilon}_{1}^{N}(t)\right\|^{2}=\left\langle g_{v}\left(u_{x}^{N}(t)\right)-g_{v}\left(u_{x}^{N-1}(t)\right), \hat{\varepsilon}_{1}^{N}(t)\right\rangle \\
& -c_{1}\left\langle\int_{0}^{t} e^{-c_{1}(t-s)}\left(g_{v}\left(u_{x}^{N}(s)\right)-g_{v}\left(u_{x}^{N-1}(s)\right)\right) d s, \hat{\varepsilon}_{1}^{N}(t)\right\rangle \\
& +\left\langle\int_{0}^{t} e^{-c_{1}(t-s)}\left(g_{i n}\left(\varepsilon_{1}^{N-1}(s)\right)-g_{i n}\left(\varepsilon_{1}^{N-2}(s)\right)\right) d s, \hat{\varepsilon}_{1}^{N}(t)\right\rangle .
\end{aligned}
$$

Using similar techniques to those above, we obtain that for some constants $\mu_{5}, \mu_{6}, \mu_{7}$ independent of $N$

$$
\left\|\hat{\varepsilon}_{1}^{N}(t)\right\|^{2} \leq \mu_{5}\left\|\hat{u}_{x}^{N}(t)\right\|^{2}+\mu_{6} \int_{0}^{t}\left\|\hat{u}_{x}^{N}(\tau)\right\|^{2} d \tau+\mu_{7} \int_{0}^{t}\left\|\varepsilon_{1}^{N-1}(\tau)-\varepsilon_{1}^{N-2}(\tau)\right\|^{2} d \tau
$$

Substituting (4.87) into (4.89) we find

$$
\left\|\hat{\varepsilon}_{1}^{N}(t)\right\|^{2} \leq \mu_{8} \int_{0}^{t}\left\|\varepsilon_{1}^{N-1}(\tau)-\varepsilon_{1}^{N-2}(\tau)\right\|^{2} d \tau
$$

which together with (4.88) yields

$$
\begin{aligned}
\left\|\hat{u}_{t}^{N}(t)\right\|^{2}+\left\|\hat{u}_{x}^{N}(t)\right\|^{2}+\left\|\hat{\varepsilon}_{1}^{N}(t)\right\|^{2} & \leq \mu \int_{0}^{t}\left\|\varepsilon_{1}^{N-1}(s)-\varepsilon_{1}^{N-2}(s)\right\|^{2} d s \\
& \leq \mu \frac{\left(\mu_{8} t_{1}\right)^{N-2}}{N-2 !}\left\|\varepsilon_{1}^{1}\right\|_{L^{2}\left(0, t_{1} ; H\right)}^{2},
\end{aligned}
$$

where $\mu=\mu_{2} \mu_{4} t_{1}+\mu_{3}+\mu_{8}$. This guarantees that $\left\{u^{N}(t)\right\},\left\{u_{x}^{N}(t)\right\},\left\{\varepsilon_{1}^{N}(t)\right\}$ are Cauchy sequences. Using the strong convergence of these sequences we can take a limit in (4.77) and (4.80) to obtain the existence of a weak solution of $(4.77)-(4.80)$ on the interval $\left[0, t_{1}\right]$. Uniqueness of the weak solution can be derived in the usual way. Now the weak solution can be extended to the intervals $\left[t_{i}, t_{i+1}\right]$ as in [6]. Thus we proved that (4.77)-(4.80) has a unique global weak solution.

Remark 4.1 It is possible to establish the global existence of a weak solution under local Lipschitz properties and growth conditions on the nonlinear functions $g_{e}, g_{v i}, g_{v d}, g_{i n}$. Taking $u_{t}^{N}$ as a test function in (4.77) and using standard inequalities we can show that the iterates $\left\{u^{N}(t)\right\},\left\{\varepsilon_{1}^{N}(t)\right\}$ are bounded by a constant, independent of $N$. Thus the computations (4.81)(4.91) can be repeated in this ball using the local Lipschitz property of the nonlinear functions.

\section{Acknowledgment}

The research of the second and third authors was supported in part by the Air Force Office of Scientific Research under grant AFOSR F49620-98-1-0180. 


\section{References}

[1] A.S. Ackleh, H.T. Banks and G.A. Pinter, On a nonlinear beam equation, Applied Math. Letters, to appear.

[2] H.T. Banks, D.S. Gilliam, and V.I. Shubov, Global solvability for damped abstract nonlinear hyperbolic systems, Differential and Integral Equations, 10 (1997), 309-332.

[3] H.T. Banks, K. Ito, and Y. Wang, Well posedness for damped second order systems with unbounded input operators, Differential and Integral Equations, 8 (1995), 587-606.

[4] H.T. Banks, N.J. Lybeck, M.J. Gaitens, B.C. Muñoz and L.C. Yanyo, Computational methods for estimation in the modeling of nonlinear elastomers, Kybernetika, 32 (1996), $526-542$.

[5] H.T. Banks, G.A. Pinter and L.K. Potter, Modeling of nonlinear hysteresis in elastomers under uniaxial tension, J. Intelligent Mat. Systems and Structures, 10 (1999), 116-134.

[6] H.T. Banks, G.A. Pinter and L.K. Potter, Existence of unique weak solutions to a dynamical system for nonlinear elastomers with hysteresis, CRSC Technical Report TR 98-43, N.C. State Univ., November, 1998; Differential and Integral Equations, 13 (2000), 1001-1024.

[7] H.T. Banks, G.A. Pinter, L.K. Potter, M.J. Gaitens and L.C. Yanyo, Modeling of quasistatic and dynamic load responses of filled viscoelastic materials, in Modeling: Case Studies from Industry, E. Cumberbatch and A. Fitt, eds., Cambridge University Press, to appear.

[8] R. Dautray and J. L. Lions, Mathematical Analysis and Numerical Methods for Science and Technology, Volume 5, Evolution Problems I, Springer, New York, 2000.

[9] J. Ha and S. Nakagiri, Existence and regularity of weak solutions for semilinear second order evolution equations, Funkcialaj Ekvacioj, 41 (1998), 1-24. 